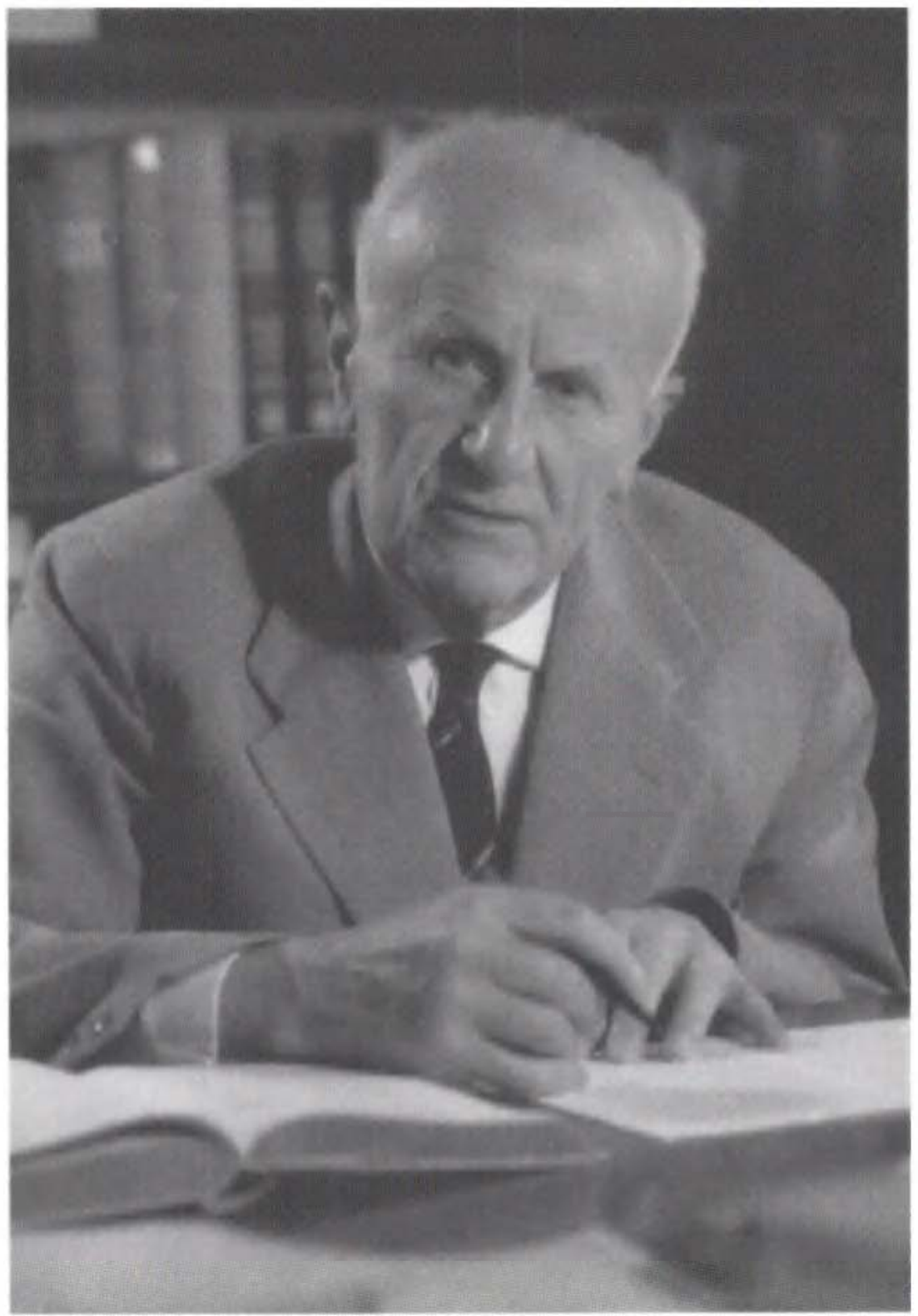

Theodor Mayer 


\section{RETO HEINZEL}

\section{THEODOR MAYER}

\section{Ein Wissenschaftsorganisator mit "großen Möglichkeiten«}

Zahlreich waren die Denkanstöße, die der in Österreich geborene Historiker Theodor Mayer der deutschen Mediävistik des vergangenen Jahrhunderts gab. Namentlich seine Beiträge zur verfassungsgeschichtlichen Forschung galten als wegweisend, und die von ihm geprägten Konzepte des frühmittelalterlichen Personenverbands, des institutionellen Flächenstaates sowie die Auffassung einer Rodungs- bzw. Königsfreiheit beeinflußten die westdeutsche Diskussion über die hochmittelalterliche Staatsentwicklung über Jahrzehnte hinweg'. Doch auch als Wissenschaftsorganisator hat er sich einen bleibenden Namen geschaffen. Der Fachwelt dürfte er zuallererst als Begründer des Konstanzer Arbeitskreises für mittelalterliche Geschichte, einem »bedeutenden Impulsgeber westdeutscher Mittelalterforschung ${ }^{2}{ }^{2}$, in Erinnerung bleiben.

Theodor Mayer war nicht an der Gründung des Deutschen Historischen Instituts in Paris im Jahre 1958 beteiligt und kann somit auch nicht zu den tatsächlichen Gründervätern gezählt werden. Gleichwohl ist seine Person mit der Geschichte bzw. Vorgeschichte des Instituts untrennbar verbunden, hatte sich Mayer doch während des Zweiten Weltkrieges dafür eingesetzt, in der französischen Hauptstadt ein deutsches historisches Forschungsinstitut zu errichten. Mayers Denkschrift vom Februar 1941 läßt sowohl seine wissenschaftlichen

' Zu den ersten Arbeiten dieser Richtung zählen Theodor MAYER, Die Stellung Rheinfrankens in der deutschen Geschichte, in: Korrespondenzblatt des Gesamtvereins der deutschen Geschichts- und Altertumsvereine 82 (1934), Nr. 1, S. 7-20 und DERS., Der Staat der Herzoge von Zähringen (Freiburger Universitätsreden, 20), Freiburg i.Br. 1935, weiter auch DERS., Die Ausbildung der Grundlagen des modernen deutschen Staates im hohen Mittelalter, in: HZ 159 (1939), S. 457-487 (der Aufsatz ist die ausgearbeitete Fassung von Mayers Vortrag für den Internationalen Historikertag in Zürich von 1938). Vgl. zur Rezeptionsgeschichte u.a. Hans K. SCHULZE, Rodungsfreiheit und Königsfreiheit, in: HZ 219 (1974), S. 529-550 und František GRAUS, Verfassungsgeschichte des Mittelalters, in: HZ 243 (1986), S. 529-589.

2 Anne Chr. NAGEL, Im Schatten des Dritten Reichs. Mittelalterforschung in der Bundesrepublik Deutschland 1945-1970, Göttingen 2005, S. 301f.; vgl. auch Thomas ZOTZ, Deutsche Mediävisten und Europa. Die Freiburger Historiker Theodor Mayer und Gerd Tellenbach im »Kriegseinsatz" und in der Nachkriegszeit, in: Bernd MARTIN (Hg.), Der Zweite Weltkrieg und seine Folgen. Ereignisse, Auswirkungen, Reflexionen, Freiburg 2006, S. 31-50. 
Absichten als auch die ideologisch-politischen Implikationen dieser Initiative deutlich werden. Die Denkschrift stellt kein Ruhmesblatt deutscher Wissenschaftsgeschichte dar. Das Schriftstück führt zudem deutlich vor Augen, wie weit die Enthemmung in den Reihen deutscher Wissenschaftler anderthalb Jahre nach Kriegsbeginn bisweilen bereits fortgeschritten war.

\section{Ausgangslage}

In der Denkschrift vom 10. Februar 1941 schlug Theodor Mayer vor, in Paris ein deutsches historisches Institut zu begründen. Dieses knapp sechseinhalb Schreibmaschinenseiten umfassende Papier markiert den Beginn einer ungefähr zweieinhalb Jahre dauernden Episode, die zu keinem konkreten Ergebnis führte. Die Gründe für das Scheitern des Institutsplans sind vielfältig. Sie lassen sich unter anderem mit dem charakteristischen Kompetenzwirrwarr im nationalsozialistischen Staat, den ministeriellen Machtkämpfen sowie mit dem aus deutscher Sicht zunehmend ungünstigen Kriegsverlauf erklären. Mayer sah sich jedenfalls wiederholt dazu gezwungen, seinen Institutsplan zu überarbeiten und den sich rasch verändernden politischen Gegebenheiten anzupassen. Noch im Juni 1942 sprach er davon, im bestehenden, kulturpolitisch tätigen Deutschen Institut in Paris einen historischen Stützpunkt einzurichten ${ }^{3}$. Die Leitung dieses Stützpunktes sollte Mayers Protegé Heinrich Büttner übernehmen, der als Mitarbeiter der Gruppe Archivwesen der deutschen Militärverwaltung mit der französischen Archivsituation bestens vertraut war ${ }^{4}$. Ein Jahr später war dann nur noch von einem Arbeitsplatz für einen Historiker die Rede $^{5}$. Schließlich konnte selbst dieser nicht realisiert werden.

4 Archiv der MGH, 537/37, Mayer an Epting vom 5.3.1942. Vgl. zu Büttner den Beitrag von Wolfgang Freund im vorliegenden Band. Vgl. zur Gruppe Archivwesen Karl Heinz ROTH, Eine höhere Form des Plünderns. Der Abschlußbericht der »Gruppe Archivwesen« der deutschen Militärverwaltung in Frankreich 1940-1944, in: 1999. Zeitschrift für Sozialgeschichte des 20. und 21. Jahrhunderts 4 (1989) 2, S. 79-112, hier S. 108. Die Gruppe bestand von 1940-1944 und wurde von Georg Schnath geleitet. Mitglieder waren durchwegs hoch qualifizierte Arbeitskräfte, die mit ihrer Tätigkeit einen Beitrag zum Kulturgutraub in den besetzten Westgebieten leisteten; vgl. Wolfgang Hans STEIN, Georg Schnath und die französischen Archive unter deutscher Besatzungsverwaltung, in: Robert KRETZSCHMAR u.a. (Hg.), Das deutsche Archivwesen und der Nationalsozialismus, Essen 2007, S. 175-194; Torsten MUSIAL, Staatsarchive im Dritten Reich. Zur Geschichte des staatlichen Archivwesens in Deutschland 1933-1945, Potsdam 1996; Anja HEUSS, Kunst- und Kulturgutraub. Eine vergleichende Studie zur Besatzungspolitik der Nationalsozialisten in Frankreich und der Sowjetunion, Heidelberg 2000, S. 161-204.

5 Archiv der MGH, 537/8, REM an Mayer vom 29.7.1943. 
Als Conrad Grau anfangs der 1990er Jahre die damaligen Ereignisse aus den Akten rekonstruierte ${ }^{6}$, bemühte er sich, die wissenschaftlichen Leistungen Mayers von dessen wissenschaftsorganisatorischer Tätigkeit zu trennen. Mayer sei bereit gewesen, resümierte Grau, sich »unter Wahrung des wissenschaftlichen Anspruchs [...] mit maßgebenden Stellen des nationalsozialistischen Staates zu arrangieren «? Grau ging hier also von zwei Sphären aus, die sich nicht berühren ${ }^{8}$. Ihm ging es in seinem Beitrag in erster Linie um die Dokumentation und den Abdruck der einschlägigen, zu jenem Zeitpunkt in Westdeutschland noch völlig unbekannten Quellen". Aus diesem Grund stellte er auch nicht die Frage nach anderweitigen Zusammenhängen.

Vierzehn Jahre nach Graus Ausführungen kommt man allerdings nicht umhin, den Wechselwirkungen zwischen Wissenschaft und Politik mit geschärfter Aufmerksamkeit zu begegnen. So gehe ich generell davon aus, daß außerwissenschaftliche Faktoren die wissenschaftliche Tätigkeit beeinflussen und damit Auswirkungen auf die Erkenntnisinteressen sowie die Formulierung von wissenschaftlichen Zielvorgaben haben ${ }^{10}$. Im Fall von Theodor Mayer stellt sich dabei die Frage nach den politischen Implikationen seiner wissenschaftsorganisatorischen Tätigkeit. Versucht man nun, die Paris-Initiative dieses umtriebigen Historikers nicht nur in den unmittelbaren, sondern in einen weiteren Entstehungszusammenhang einzuordnen, wird man den Blick auf Mayers Tätigkeit vor 1941 richten müssen. Dabei sollen folgende Fragen leitend sein: Wie läßt sich der Plan eines Pariser Instituts mit Mayers allgemeinem Wissenschaftsverständnis sowie mit seinen allgemeinen wissenschaftsorganisatorischen Vorstellungen und Absichten in Verbindung bringen? Warum stellte Mayer diese kulturpolitischen Überlegungen an bzw. welche wissenschaftspolitischen Überzeugungen spiegeln sich in dieser Initiative?

Um Antworten auf diese Fragen zu finden, werde ich im folgenden meinen Blick auf Mayers Aktivitäten der 1930er Jahre richten. Grundlage ist die Denkschrift aus dem Jahre 1941, die mir zugleich als zentraler Bezugspunkt dienen wird.

- Conrad GraU, Planungen für ein Deutsches Historisches Institut in Paris während des zweiten Weltkrieges, in: Francia 19/3 (1993), S. 109-128.

Ibid., S. 110.

Ibid., S. 117f.

Als Mayer 1944 die Evakuierung der MGH von Berlin nach Pommersfelden veranlaßte, verblieb ein Teil der Institutsakten bei der Dienststelle in Berlin. Nach dem Krieg gelangte dieser Teil der MGH-Akten ins Archiv der ehemaligen Preußischen Akademie der Wissenschaften in Ostberlin. Heute befinden sie sich im Archiv der MGH in München.

10 Hier beziehe ich mich auf Ludwik FLECK, Entstehung und Entwicklung einer wissenschaftlichen Tatsache. Einführung in die Lehre vom Denkstil und Denkkollektiv, Frankfurt a.M. ${ }^{4} 1999$, S. 90. 
Die militärischen Erfolge der Wehrmacht im Mai und Juni 1940 hatten weite Teile der deutschen Bevölkerung in einen wahren Freudentaumel versetzt. Wie die meisten seiner Berufskollegen geriet auch Theodor Mayer in einen Rausch nationaler Hochstimmung. Die wissenschaftliche Tätigkeit empfand er jetzt als eine doppelte Freude. Und wenn er auch beklagte, das Kriegshandwerk den Jüngeren überlassen zu müssen, war er doch überzeugt, als Wissenschaftler einen kriegswichtigen Beitrag leisten zu können ${ }^{11}$.

Nachdem das Deutsche Reich im Mai/Juni 1940 Frankreich bezwungen und damit die kontinentaleuropäische Vorherrschaft errungen hatte, sah Mayer das Ende des Krieges in greifbare Nähe gerückt. Unter diesem Eindruck begann er, konkrete Überlegungen zur Neuordnung Europas in der vermeintlich unmittelbar bevorstehenden Nachkriegszeit anzustellen. Diesbezüglich sah er nicht nur die Politik, sondern namentlich die Geschichtswissenschaft vor groBe Herausforderungen gestellt. In seiner Denkschrift von 1941 bezeichnete er die Erreichung einer der politischen Stellung entsprechenden Führerfunktion im europäischen Raum als eine der wichtigsten Aufgaben der deutschen Geschichtswissenschaft ${ }^{12}$. Die Konzeption eines europäischen Geschichtsbildes, das wesentlich von der deutschen Geschichtswissenschaft aus bestimmt sein sollte, erachtete der Historiker als derart wichtig, daß er für dieses »letzte Ziel«, wie er es nannte, im Reichserziehungsministerium beharrlich die Werbetrommel rührte ${ }^{13}$. Mayers Interpretation fußte dabei auf der festen Überzeugung, daß Europa nach dem militärischen ein geistiger Kampf bevorstehen werde $^{14}$. Wollte die deutsche Geschichtswissenschaft diesen für sich entscheiden und damit die geistige Führung in Europa übernehmen, dann war es unumgänglich, im besetzten Ausland wissenschaftliche Stützpunkte einzurichten. Dies betraf im Besonderen den "Erbfeind « Frankreich, gegen dessen politische Ansprüche Mayer bereits als Leiter der Westdeutschen Forschungsgemeinschaft in den 1930er Jahren agiert hatte. Jedenfalls werde man, schrieb Mayer seinem Kontaktmann Werner Best, damals Leiter der Abteilung Verwaltung beim deutschen Militärbefehlshaber in Frankreich, um die Errichtung einer rein wissenschaftlichen Forschungsstätte in Paris nicht herumkommen ${ }^{15}$.

Mayer war der Ansicht, daß die von ihm formulierte Nachkriegsaufgabe nur durch strengste wissenschaftliche Arbeit auf weitester Grundlage und mit den

"Stadtarchiv Konstanz (StdAK), NL Mayer, Varia 24, Mayer an Bock vom 3.6.1940.

12 Archiv der MGH, 537/61, Denkschrift über die Errichtung eines deutschen historischen Instituts in Paris vom 10.2.1941.

13 Vgl. z. B. StdAK, NL Mayer, 13/67a, Mayer an Klett (REM) vom 11.2.1941.

14 Archiv der MGH, 537/61, Denkschrift über die Errichtung eines deutschen historischen Instituts in Paris vom 10.2.1941.

$15 \mathrm{Vgl}$. auch Archiv der MGH, 537/49, Mayer an Best vom 1.7.1941. 
besten Kräften und Methoden aber auch der klarsten Zielsetzung gelöst werden könne ${ }^{16}$. Mit der Forderung nach strengster Wissenschaftlichkeit in der Durchführung postulierte Mayer eine scharfe Trennlinie zwischen Wissenschaft und Propaganda. Diese Grenze aufrecht zu erhalten, entsprach seinem Ethos als ernsthafter Wissenschaftler - eine Haltung, die er mit der großen Mehrheit seiner deutschen Berufskollegen teilte.

Allerdings ist nicht $z u$ übersehen, daß die wissenschaftliche Wahrnehmung Theodor Mayers mit seinem nationalpolitischen Bewußtsein eng gekoppelt war. So stand für ihn unverrückbar fest, daß die deutsche Geschichtsforschung der französischen weit überlegen sei. Zwar wies Mayer auf die ruhmreiche Vergangenheit der französischen Geschichtswissenschaft hin, doch in seinen Augen hatte die deutsche Historiographie seit dem Weltkrieg einen solchen Aufschwung in der Methode der Forschung und in den Problemstellungen vollzogen, daß sie »heute die französische zweifellos überholt« habe. Konkret lag für Mayer diese Überlegenheit in der methodischen Verbindung zwischen Urkundenforschung und geschichtlicher Landesforschung begründet ${ }^{17}$, deren Möglichkeiten er selbst seit längerem auslotete. Die deutsche Geschichtswissenschaft schien ihm jedenfalls heute wohl in der Lage zu sein, sich eine führende Stellung in der europäischen Geschichtswissenschaft zu sichern ${ }^{18}$.

Wenn Theodor Mayer die Thesen eines Franz Petri zur Entstehung der germanischen Sprachgrenze in Nordfrankreich ${ }^{19}$ auch nicht in ihrer ganzen Schärfe teilte - die Vorstellung einer geschlossenen germanischen Siedlung im Westraum hatte es ihm zumindest angetan ${ }^{20}$. So schrieb Mayer, die neuere (deutsche) Geschichtsforschung habe den Nachweis erbracht, daß Nordfrankreich ein weitgehend "germanischer Raum« sei. Mochte auch der größte Teil der Germanen dort sprachlich romanisiert worden sein - für Mayer stand auBer Frage, daß wdem Blute und den großen geschichtlichen Leistungen auf allen Gebieten des ganzen Lebens in Volk und Staat nach [...] der Anteil der Germanen bedeutend" gewesen sein müsse. Der Nachweis sollte nun im einzelnen genau und verläßlich geführt werden. Da man sich methodisch im Vor-

16 Archiv der MGH, 537/61, Denkschrift über die Errichtung eines deutschen historischen Instituts in Paris vom 10.2.1941.

17 Darüber hinaus sei auch die man und für sich reichere und mannigfaltigere Geschichte Deutschlands a für den Aufschwung verantwortlich; Archiv der MGH, 537/62, Denkschrift über die Errichtung eines deutschen historischen Instituts in Paris vom 10.2.1941.

8 Ibid

19 Franz PETRI, Germanisches Volkserbe in Wallonien und Nordfrankreich. Die fränkische Landnahme in Frankreich und den Niederlanden und die Bildung der westlichen Sprachgrenze, 2 Bde., Bonn 1937.

20 Für den »Westraum«, so Mayer, hätten Petri und Steinbach »den Nachweis einer unter germanischer Führung stehenden, durch germanische Siedlung und Herrschaft begründeten Kultureinheit von der Loire bis zur Ostsee erbracht«; Theodor MAYER, Wirtschaftsund Siedlungsgeschichte, in: Deutsche Wissenschaft. Arbeit und Aufgabe, Leipzig 1939, S. 26-28, hier S. 27. 
teil wähnte, war Mayer überzeugt, auf dieser Grundlage »ein[en] allgemeine[n] deutsche[n] Führungsanspruch« begründen zu können ${ }^{2 !}$.

\section{Geschichtswissenschaft mit Ziel}

Wenn Mayer in seiner Denkschrift von einer Führerfunktion sprach, geschah das nicht aus opportunistischen Gründen. Schaut man sich Mayers wissenschaftlichen Werdegang genauer an, so zeigt sich, daß sich in seiner wissenschaftsorganisatorischen Initiative Denkfiguren finden, deren Ursprünge in den 1930er Jahren liegen. So hatte sich Mayer bereits damals zu einer an den politischen Bedürfnissen orientierten Geschichtsschreibung bekannt: Für seine Disziplin forderte er eine große »Aufgabe und Funktion«, die er - prägnant und zeitgemäß - als »Führeramt im Volksganzen« bezeichnete ${ }^{22}$. Welche wissenschaftsorganisatorischen Vorstellungen in dieser Aussage zum Ausdruck gelangten, soll im folgenden erläutert werden.

Als Theodor Mayer im Jahre 1930 von Prag nach Gießen übersiedelte, verschoben sich auch seine wissenschaftlichen Interessen und die damit verknüpften räumlichen Bezugspunkte. Er wandte sich nun definitiv von den wirtschaftsgeschichtlichen Problemen ab und dafür der hochmittelalterlichen Verfassungsgeschichte zu. Dabei konzentrierte er sich zunächst auf die westdeutschen regionalen »Räume«, die er in Beziehung zur Reichs- und Volksgeschichte setzte. Mayers Ansichten waren dabei stark von der gesamtdeutschen Geschichtsauffassung geprägt ${ }^{23}$. Dieses vom österreichischen Historiker Heinrich von Srbik begründete Konzept war als Alternative zur groß- und kleindeutschen Auffassung entwickelt worden, wobei in Anlehnung an den alten Reichsgedanken das Gemeinsame zwischen Deutschland und Österreich betont wurde. Durch den Bezug auf das Gesamtdeutschtum sollte eine einheitliche Geschichte s)des durch Blut, Sprache und Kultur, durch Raum und Schicksal einheitlichen deutschen Volkes « herausgearbeitet und damit das Trennende endgültig überwunden werden ${ }^{24}$. Der gesamtdeutschen Auffassung lag sowohl ein wissenschaftliches als auch ein nationalpolitisches Ziel zugrunde ${ }^{25}$.

21 Archiv der MGH, 537/62-63, Denkschrift über die Errichtung eines deutschen historischen Instituts in Paris vom 10.2.1941.

22 Theodor MAYER, Vortrag anläßlich der Festsitzung der Badischen Historischen Kommission vom 14.12.1935, in: ZGOR 49 (1936), S. 523-536, hier S. 531.

23 Über diese Zusammenhänge siehe MAYER, Stellung Rheinfrankens (wie Anm. 1), S. $7 \mathrm{f}$.

${ }^{24}$ Heinrich VON SRBIK, Gesamtdeutsche Geschichtsauffassung, in: Deutsche Vierteljahrsschrift für Literaturwissenschaft und Geistesgeschichte 8 (1930), S. 1-12, hier S. 2, 10.

25 Vgl. auch Matthias PAPE, Ungleiche Brüder. Österreich und Deutschland 1945-1965, Köln 2000, S. 165. 
Mayer ging davon aus, daß Landesgeschichte und Reichsgeschichte untrennbar miteinander verbunden sind. In seiner Freiburger Antrittsvorlesung von 1934 stellte er diesen Prozeß der Wechselwirkung dar, zugleich beschrieb er die Begriffe "Landschaft«, "Staat" und "Volk« als untrennbare Einheit. Damit postulierte er in politisch aktualisierender Weise einen Zusammenhang zwischen mittelalterlichem und »Drittem Reich «, zu jener Zeit bekanntlich ein äußerst populärer Gedanke ${ }^{26}$. Für Mayer lag der Ausgangspunkt des nationalsozialistischen Staates im "Volk«, das er »in unmittelbarem Verhältnis « zum »Führer« $\operatorname{sah}^{27}$. Er war der festen Überzeugung, daß jede Gegenwart der $\mathrm{Ge}-$ schichtswissenschaft ganz bestimmte Aufgaben stelle, die, insofern sie die Aufgaben als solche erkennt, zu einer »unbedingt aktuellen Wissenschaft» werde. Durch den unbedingten Zusammenhang zwischen Volk und Führerstaat werde die Geschichte des Volkes zur »Volksgeschichte schlechthin, Staats- und Volksgeschichte«, hielt Mayer fest, »sind so betrachtet auch in der Forschung nicht mehr zu trennen ${ }^{28}$.

Die wissenschaftliche Erforschung des deutschen Volkes war für den gebürtigen Österreicher bereits Ende der 1920er Jahre zum Thema geworden. Unter dem Eindruck der Forschungen im Umfeld der Stiftung für Volks- und Kulturbodenforschung in Leipzig begann Mayer damals, sich mit Fragen des "Volkstums« und des »Raumes« auseinanderzusetzen ${ }^{29}$, Themen, die ihn nach seiner Übersiedlung nach Gießen im Jahre 1930 noch intensiver beschäftigten. In Freiburg, wohin er 1934 berufen wurde, fand Mayer dann ein Forschungsumfeld vor, das gerade im Bereich der Landes- und Volksforschung ungemein lebendig war und den mittlerweile 50jährigen Mediävisten zu neuen wissenschaftlichen Fragestellungen anregte. Auch in wissenschaftsorganisatorischer Hinsicht waren die Freiburger Jahre von nachhaltiger Bedeutung, denn Mayer kam in dieser Zeit mit verschiedenen wissenschaftlichen Institutionen in Kontakt. Institutionen, deren Leitung er in dieser Zeit ausübte und auf die er entscheidend einzuwirken verstand.

An erster Stelle ist hier die Westdeutsche Forschungsgemeinschaft (WFG) zu nennen, deren Leitung Mayer 1935 übernahm. Diese interdisziplinär ausgerichtete Gemeinschaft gleich gesinnter Forscher war 1931 gegründet worden. Sie zählte damals mit vier weiteren, regional unterschiedlich ausgerichteten Forschungseinrichtungen zum Verbund der sogenannten Volksdeutschen For-

26 Vg1. dazu allgemein Ursula WoLf, Litteris et Patriae. Das Janusgesicht der Historie, Frankfurt a.M. 1997.

27 Theodor MAYER, Volk und Staat der Deutschen. Vom Ersten, Zweiten und Dritten Reich, in: Glaube und Volk in der Entscheidung 4 (1935) 3, S. 60-72, hier S. 70 f.

28 MAYER, Staat der Herzoge von Zähringen (wie Anm. 1), S. 3 f., 31.

29 DERS., Aufgaben der Siedlungsgeschichte in den Sudetenländern, in: Deutsche Hefte für Volks- und Kulturbodenforschung 1 (1931) 3, S. 129-151. Dieser Aufsatz wurde zwar erst 1931 gedruckt, er entstand aber bereits während der Zeit in Prag, wo Mayer von 1923-1930 an der Deutschen Universität lehrte. 
schungsgemeinschaften (VFG) ${ }^{30}$. Wie ihre Vorgängerin, die Stiftung für Volks- und Kulturbodenforschung in Leipzig, konzentrierten sich auch die VFG ganz auf die interdisziplinäre Erforschung von deutsch besiedelten Grenz- und Sprachräumen. Im Falle der WFG dominierte eine antifranzösische Stoßrichtung. Zwischen 1935 und 1939 organisierte Theodor Mayer zahlreiche wissenschaftliche Tagungen, auf denen sich ein geladener Teilnehmerkreis jeweils mit einem ganz bestimmten Thema aus dem westdeutschen Grenzraum befaßte. Wie politisch die an den Methoden der Landes- und Volksforschung orientierte Gemeinschaftsarbeit der WFG war, wird aus den zahlreichen Tagungsprotokollen ersichtlich, die allesamt als streng vertraulich zu behandeln waren ${ }^{3 !}$. Daß Theodor Mayer wiederholt auf den gesamtdeutschen Charakter der Westforschung hingewiesen hat, macht deutlich, daß er Landesgeschichte, Volkstumsforschung und gesamtdeutsche Geschichtsbetrachtung in einem direkten Zusammenhang $\mathrm{sah}^{32}$.

Der WFG waren verschiedene landeskundliche Institute angeschlossen. Sie verfügte somit über zahlreiche kooperierende Forschungseinrichtungen und Kontaktstellen und war für die Organisation und Koordination der volkstumswissenschaftlichen Forschungen in den angeschlossenen Instituten zustän$\operatorname{dig}^{33}$. Eines dieser Institute war das Alemannische Institut in Freiburg ${ }^{34} .1931$ nach dem Vorbild des Bonner Instituts für geschichtliche Landeskunde der Rheinlande $^{35}$ gegründet, wurde es im Zuge der nationalsozialistischen »Machtergreifung« aufgelöst und neu organisiert. Im Frühjahr 1935 wurde

30 Vgl. zur Geschichte der VFG Michael FAHLBUSCH, Wissenschaft im Dienst der nationalsozialistischen Politik? Die "Volksdeutschen Forschungsgemeinschaften" von 19311945, Baden-Baden 1999.

31 Vgl. zum Politisierungsgrad der WFG auch Peter SCHÖTTLER, Die historische "Westforschung " zwischen "Abwehrkampf" und territorialer Offensive, in: DERS. (Hg.), Geschichtsschreibung als Legitimationswissenschaft 1918-1945, Frankfurt a.M. 1997, S. 204-261.

32 Z.B. im Jahresbericht der WFG für 1935/36; Bundesarchiv Berlin-Lichterfelde (BABL), R153/1495, Jahresbericht der Westdeutschen Forschungsgemeinschaft für 1935/36 vom 18.3.1936.

${ }^{33}$ FaHLBUSCH, Wissenschaft (wie Anm. 30), S. 368.

34 "Darüber hinaus ist das Alemannische Institut in das System der Forschungsgemeinschaften einzugliedern, d.h. es bildet zusammen mit der bereits bestehenden Rheinischen Forschungsgemeinschaft und dem Elsaß-Lothringischen Institut in Frankfurt a.M. die Westdeutsche Forschungsgemeinschaft«; Stadtarchiv Freiburg (StdAF), C4/X/19/10, Reichsministerium des Innern an Kerber vom 21.10.1935. Nebst dem Oberrheinischen Institut gehörten zur WFG das Institut für geschichtliche Landeskunde der Rheinlande an der Universität Bonn, das Elsaß-Lothringen-Institut an der Universität Frankfurt am Main sowie das Provinzialinstitut für Westfälische Landes- und Volksforschung in Münster; Politisches Archiv des Auswärtigen Amtes (PA/AA), R60293, Jahresbericht der Westdeutschen Forschungsgemeinschaft für 1936/37, B1. E63168.

35 Vgl. Marlene NiKOLAY-PANTER, Geschichte, Methode, Politik. Das Institut für geschichtliche Landeskunde der Rheinlande 1920-1945, in: Rheinische Vierteljahrsblätter (1996), S. 233-262. 
Theodor Mayer zum wissenschaftlichen Leiter ernannt. Organisatorisch und personell war das Alemannische Institut eng mit der WFG verflochten, und Mayer beabsichtigte, diese städtische Einrichtung auf eine neue Grundlage zu stellen $^{36}$. Unter Anwendung moderner landesgeschichtlicher Methoden sollte jetzt die wissenschaftliche Erforschung des »alemannischen Raumes« vorangetrieben werden ${ }^{37}$. Damit gerieten auch jene Gebiete in den wissenschaftlichen Fokus, die jenseits der deutschen Grenze lagen.

$\mathrm{Zu}$ diesem Zweck beabsichtigte Theodor Mayer, die Zusammenarbeit mit ausländischen Forschern, namentlich mit Schweizern und Elsässern, zu verstärken - eine Initiative, die unter den damaligen politischen Bedingungen rasch zu einer kulturpolitisch brisanten Angelegenheit werden konnte. Dem Historiker war es ein wichtiges Anliegen, möglichst viele ausländische Forscher mit den methodischen Standards der deutschen Landesforschung vertraut zu machen und von deren wissenschaftlichem Potential und Bedeutung zu überzeugen. Dabei war sich Mayer bewußt, daß »wir nur durch strengste wissenschaftliche Leistung auf die Notwendigkeit der Zusammenarbeit mit der deutschen Wissenschaft verweisen können ${ }^{38}$.

Der Erfolg dieser Strategie hing in entscheidendem Maße davon ab, ob es gelang, die im Ausland teils bestehenden Vorbehalte gegenüber deutschen kulturpolitischen Initiativen zu zerstreuen. Aus diesem Grund setzte sich Mayer beharrlich für eine Namensänderung des Alemannischen Instituts ein, da er vermutete, daß sübergroße Empfindlichkeiten des Auslandes bestünden und man mit dem Wort "alemannisch « Absichten politischer Annexion ${ }^{39}$ assoziieren könnte. Anfang 1936 wurde das Institut in Oberrheinisches Institut für geschichtliche Landeskunde umbenannt ${ }^{40}$. Gemäß Mayer entsprach der neue Name zudem »besser den Aufgaben, die nicht ausschließlich auf den oberrheinischen Raum beschränkt sein, sondern allgemein die Fragen behandeln sollen, die sich aus der Berührung deutschen und germanischen Volkstums mit nicht germanischem im deutschen Südwestraum ergeben ${ }^{41}$.

In der Folge bemühte sich Mayer allerdings vor allem um Kontakte zu jenen Gelehrten, welche »die gleichen wissenschaftlichen Ziele verfolgen wie das Institut ${ }^{42}$. In der Praxis bedeutete dies, daß ein wissenschaftlicher Austausch nur mit deutschfreundlichen Wissenschaftlern stattfand. Diese Einschätzung gilt folglich auch für die WFG, in deren Namen das Oberrheinische

$36 \mathrm{StdAF}, \mathrm{C} 4 / \mathrm{X} / 19 / 10$, Hefele an Kerber vom 26.4.1935.

37 Ibid., Satzung des Alemannischen Instituts Freiburg i.Br. (Abschrift).

38 BABL, R153/1495, Jahresbericht der Westdeutschen Forschungsgemeinschaft für 1935/36 vom 18.3.1936, Bl. 39 .

39 StdAF, C4/X/19/10, RMdI (Bourwieg) an Kerber vom 6.2.1936 sowie Eingabe Mayers an das RMdI vom 9.1.1936.

40 Ibid., Mayer an Kerber vom 9.1.1936.

41 Ibid., Eingabe Mayers an das RMdI vom 9.1.1936.

42 Ibid., Haushaltsplan für das Rechnungsjahr 1936/37 vom 10.3.1936. 
Institut mehrere Tagungen organisierte. In der damaligen politischen Situation bestand wenig Aussicht, sich unter den dem Nationalsozialismus kritisch eingestellten Ausländern Gehör zu verschaffen. Deshalb erstaunt es auch nicht, $\mathrm{da}$ es sich bei den ausländischen Tagungsteilnehmern der WFG in aller Regel um germanophile Wissenschaftler handelte. Unter den gegebenen Umständen waren vor allem sie für die interdisziplinären Methoden der deutschen Landesund Volksforschung zu gewinnen. Daß damals ein Wissenschaftler aus Unbedachtheit eine Einladung zu einer WFG-Tagung annahm, war höchst unwahrscheinlich. So lehnte auch der Schweizer Historiker Marcel Beck das Angebot zur Teilnahme an der Schönauer Tagung der WFG 1938 mit der Begründung $\mathrm{ab}$, daß die "politischen Ereignisse der letzten Zeit [...] einem Schweizer größte Zurückhaltung gegenüber jeglicher volksdeutscher Aufbauarbeit auf[erlegen, RH], wie sie in der W.F. [WFG, RH] vornehmlich getrieben wird ${ }^{43}$. Das Ergebnis dieser Form von Wissenschaftspolitik war eindeutig: Kontroverse Diskussionen über Methoden, geschweige denn über Erkenntnisinteressen blieben aus ${ }^{44}$.

Als Vorsitzender der WFG formulierte Theodor Mayer das ambitionierte Ziel einer »neuen Grundlegung der alemannischen Geschichte«. Er wollte dies mit "umfassende[n] Forschungen auf allen Gebieten « erreichen und rechnete mit einem raschen Abschluß innert zweier Jahre ${ }^{45}$. Zur »neuen Grundlegung« sollte auch die Universität Freiburg ihren Beitrag leisten. Anläßlich der anstehenden Neubesetzung des konkordatsgebundenen Lehrstuhls für Geschichte ${ }^{46}$ präzisierte Theodor Mayer seine Vorstellungen. Die Aufgabe der Grenzuniversität sah er darin, süber die Grenze hinweg die Zusammenhänge mit dem geistigen Leben besonders der deutschen Volksteile in der Schweiz und in Elsaß-Lothringen lebendig zu erhalten und in diesem geistigen Leben sich eine führende Rolle $\mathrm{zu}$ erwerben und $\mathrm{zu}$ bewahren«. $\mathrm{Zu}$ den wichtigsten Fächern

Zentralbibliothek Zürich (ZBZ), Nachlaß Hans Nabholz, 15.11, Beck an Mayer vom 22.2.1938 (Abschrift). Marcel Beck wußte, wovon er sprach, nahm er doch ab Oktober 1935 während anderthalb Jahren einen Forschungsauftrag am Oberrheinischen Institut wahr (StdAF, C4/X/19/10, Jahresbericht des Oberrheinischen Instituts für geschichtliche Landeskunde 1936/37, o. D., S. 7).

$44 \mathrm{Vgl}$. auch die Erinnerungen von Paul Roth, dem damaligen Leiter des Staatsarchivs Basel: »Bald darauf aber erwies sich, [...] daß bei den Deutschen im Hintergrunde Kräfte im Spiele waren, die einerseits auf den Ausschluß der Straßburger Universitätslehrer abzielten und andrerseits die Gruppierung einer elsässischen und schweizerischen Gefolgschaft bezweckten« (Staatsarchiv Basel-Stadt [SABS], PA 454a, 16/3: Privatarchive, Nachlaß Paul Roth, Leiter des Staatsarchivs Basel, 1933-1956 [Erinnerungen], S. 44). Roth äußerte sich hier vor allem zu den Tagungen der WFG 1937, insbesondere nach der Tagung in Basel am 26.9.1937. Für diesen Quellenhinweis danke ich Wolfgang Freund.

45 BABL, R153/1495, Jahresbericht der Westdeutschen Forschungsgemeinschaft für 1935/36 vom 18.3.1936, B1. 40.

46 Dabei handelte es sich um den Lehrstuhl für mittlere und neuere Geschichte (Nachfolge von Philipp Funk). 
für diese Aufgabe gehörte nach Mayer die Geschichtswissenschaft, insbesondere jene Zweige, die wjenseits der Grenze nicht oder nicht voll betrieben werden«, wie er sich ausdrückte. Dazu zählte er die historischen Hilfswissenschaften und die geschichtliche Landesforschung, mit denen der "Einbau der Geschichte des Oberrheingebietes in die gesamtdeutsche Geschichte und die Eingliederung der ganzen Geschichtsforschung im Oberrheingebiet in die deutsche Geschichtswissenschaft gelingen sollte. Eine führende Stellung der deutschen Forschung war in Mayers Augen aber nur zu erreichen, wenn die Freiburger die Gebenden sind, wenn sie etwas bringen, was jenseits der Grenze nicht oder nicht genügend betrieben wird « ${ }^{47}$.

Als Publikationsort schwebte Mayer die »Zeitschrift für die Geschichte des Oberrheins « (ZGOR) vor, das Organ der Badischen Historischen Kommission (BHK), deren Vorsitzender Mayer im Mai 1935 geworden war. Aufgrund des geltenden Führerprinzips konnte er nun den Inhalt dieser wichtigen südwestdeutschen Zeitschrift für Landesgeschichte maßgeblich mitbestimmen. Die ZGOR erachtete Mayer als ideales Mittel zur Propagierung der Landes- und Volksforschung. Man brauche keine neue Zeitschrift zu gründen, meinte er, sondern es könne die ZGOR, die sowohl im Elsaß als auch in der Schweiz bekannt sei, noch "stärker auf diese Fragen eingestellt werden « ${ }^{48}$. Tatsächlich war Mayer bestrebt, möglichst viele Beiträge aufzunehmen, die sich mit der Schweiz oder aber mit der Geschichte des elsässisch-lothringischen Raumes befaßten ${ }^{49}$. Damit sollte einerseits deutsche wissenschaftliche Präsenz markiert werden, anderseits umschrieb Mayer das wissenschaftliche Leben im Elsaß generell als »zu schwach«. Er war darum überzeugt, »bei gewissen Fragen eingreifen « zu müssen ${ }^{50}$. Bei alledem stand außer Frage, daß die Beiträge von höchster wissenschaftlicher Qualität sein mußten, um der französischen Kritik möglichst wenig Angriffsflächen zu bieten ${ }^{51}$.

Die Badische Historische Kommission selbst wollte Mayer auf eine größere Anzahl von Ausschüssen verteilen. Dabei hielt er es für geboten, „daß der Vorsitzer sich, wenn er es für nötig hält, in jeden Ausschuß einschalten [...] oder ein anderes Vorstandsmitglied [...] dazu delegieren kann « ${ }^{52}$. Weil die Kommission jetzt nach dem Führerprinzip eingerichtet sei, könne er machen, was er für gut halte, schrieb Mayer Ende 1935 an den befreundeten Wiener

47 Staatsarchiv Freiburg (SAF), C 25/2, Nr. 59, Mayer an Maurer (Dekan) vom 16.1.1938.

48 BABL, R153/1495, Jahresbericht der Westdeutschen Forschungsgemeinschaft für 1935/36 vom 18.3.1936, Bl. 40.

49 Tbid; Generallandesarchiv Karlsruhe (GLAK), 449/233, Mayer an Baier vom 4.2.1936.

so Ibid., Mayer an Baier vom 5.9.1937.

51 Ibid., Mayer an Baier vom 25.3.1936.

52 Ibid., Mayer an Baier vom 24.11.1935. Über Mayers Unzufriedenheit mit der Tätigkeit der Unterausschüsse vgl. ibid., Mayer an Baier vom 14.4.1936 sowie vom 1.3.1936. In letzterem Brief liest man: »Haben Sie einmal von irgendeinem Unterausschuß etwas gehört? Die scheinen allgemein das Führerprinzip dahin aufzufassen, daß sie jetzt gar nichts mehr tun«. 
Historiker Wilhelm Bauer: »[1]ch kann über die ganzen Kredite verfügen, das Arbeitsprogramm bestimmen usw., das gibt natürlich große Verantwortung und sicher Kritik, aber man kann auch was auf die Beine bringen ${ }^{53}$.

In diesen politisierten Wissenschaftskontext gehört auch Mayers Idee einer Konzentration der bestehenden südwestdeutschen wissenschaftlichen Institutionen. Gemäß seinen Vorstellungen sollte die BHK nämlich zu einer Art Dachorganisation der südwestdeutschen Historiker werden. "Politik des historischen Mittelpunktes« nannte Mayer diesen Plan, den er in einem Brief an Hermann Baier, damals Direktor des Generallandesarchivs Karlsruhe und Schriftführer der Kommission, genauer umschrieb: »Der Sinn wäre der, daß wir alle südwestdeutschen Historiker in irgendeiner Form an die badische Kommission heranziehen würden und sozusagen der kommissionelle Mittelpunkt für Südwestdeutschland würden ${ }^{54}$. Mayer war aufgefallen, daß es in der Region keine zweite Kommission gab, deren Konkurrenz man dabei zu fürchten brauchte ${ }^{55}$. Durch persönliche Bindungen sollte die BHK in der südwestdeutschen Wissenschaftslandschaft zukünftig fester verankert werden. Darum ernannte Mayer eine ansehnliche Zahl an neuen Kommissionsmitgliedern. In manchen Fällen waren diese Ernennungsvorschläge nicht frei von politischen Erwägungen. So sollte beispielsweise der Österreicher Heinrich von Srbik Ehrenmitglied werden, obschon sich dieser keine besonderen Verdienste um die badische Geschichte (wie in den Statuten eigentlich vorgesehen) erworben hatte ${ }^{56}$. Allerdings handelte es sich bei Srbik bekanntlich um einen der prominentesten Vertreter der gesamtdeutschen Geschichtsauffassung. Dies war kein Zufall und kann als Versuch gelesen werden, die Verbindung zwischen geschichtlicher Landesforschung und gesamtdeutscher Geschichtsauffassung in personeller Hinsicht zu intensivieren.

\section{Ordnung und Ausweitung des Programms nach Kriegsbeginn}

Theodor Mayers Plan, die südwestdeutsche Landesforschung auf eine neue Grundlage zu stellen, schien auf guten Wegen zu sein. Doch zogen nicht alle der Beteiligten am selben Strick. Im Laufe der Zeit kam es zu vielfältigen und

53 Österreichische Akademie der Wissenschaften in Wien (ÖAdW), NL Wilhelm Bauer, 4/17, Mayer an Bauer vom 15.12.1935.

${ }^{54}$ Diese Politik konnte nicht zuletzt im Rahmen einer regelmäßigen Zusammenkunft der südwestdeutschen Vereine umgesetzt werden (vgl. als Beispiel GLAK, NL Andreas, $69 \mathrm{~N} / 778$, Protokoll der Zusammenkunft südwestdeutscher Geschichtsvereine vom 9./10.7.1938).

55 Ibid., 449/14, Mayer an Baier vom 5.12.1935.

56 ÖAdW, NL Wilhelm Bauer, 4/17, Mayer an Bauer vom 5.6.1935. 
letztlich unlösbaren Konflikten Mayers mit staatlichen Stellen und mit verschiedenen Wissenschaftlerkollegen. In diesen Machtkämpfen, die an dieser Stelle nicht im Detail ausgebreitet werden können, spielten wissenschaftliche, aber auch machtpolitische und nicht zuletzt persönliche Motive eine Rolle. Mayer folgte der Berufung nach Marburg 1938 jedenfalls nicht ganz freiwillig. Dort hatte er spätestens mit der Übertragung des Rektorats im Herbst 1939 wieder eine universitär wie auch gesellschaftlich exponierte Stellung inne ${ }^{57}$. Wie bereits angedeutet, versetzte die just in jener Zeit beginnende Phase der sogenannten Blitzkriege Theodor Mayer in einen wahren Freudentaumel. Dies blieb nicht ohne Auswirkungen auf die wissenschaftliche Urteilsbildung.

Diese Entwicklung läßt sich am Beispiel von Mayers Rede anläßlich der Feier der Reichsgründung und der Machtübernahme am 30. Januar 1940 veranschaulichen. In diesem Vortrag machte sich der Historiker Gedanken zur gegenwärtigen nationalsozialistischen Neuordnung Europas. Zudem skizzierte er die zukünftigen Aufgaben seiner Fachdisziplin. Ausgangspunkt seiner Überlegungen war noch immer die gesamtdeutsche Geschichtsauffassung, die Mayer nun als »Ruhmestitel der deutschen Geschichtswissenschaft als einer politischen Wissenschaft « bezeichnete. Die gesamtdeutsche Auffassung, stellte der Historiker mit Genugtuung fest, habe sich heute nals selbstverständlich durchgesetzt«. Ohne von ihrem wissenschaftlichen Charakter jemals etwas einzubüßen, habe sie dort eine Einheit geschaffen, wo die einzelstaatliche Betrachtungsweise zuvor "zerteilt und aufgelöst « habe. Das deutsche Volk, das Mayer jetzt als "selbstverständliche und unverrückbare Einheit« beschrieb, wurde als eigentlicher Ausgangs- und Fixpunkt dieser Auffassung präsentiert. Durch diesen Bezug bestand laut Mayer nicht die Gefahr, sich »in einem überund unnationalen Humanitätsideal zu verlieren « ${ }^{58}$.

Angesichts der Umwälzungen im Zuge der nationalsozialistischen Eroberungspolitik sah Mayer nun allerdings die Zeit gekommen, die "gesamtdeutsche« durch eine "gesamteuropäische Geschichtsbetrachtung" zu ersetzen. Das Ziel, das mit einem solchen Perspektivenwechsel verbunden war, lag in einer auf dem volkstumswissenschaftlichen Paradigma fußenden Art der >Beweisführung`, wonach Deutschland in der europäischen Geschichte stets die hervorragende »Ordnungsmacht « gewesen war. Nur im Rahmen der gesamteuropäischen Geschichtsbetrachtung, argumentierte Mayer, werde »die Stellung und Funktion der germanischen Mitte in Europa als das Prinzip der Ordnung und des Aufbaues verständlich ${ }^{59}$. Derartige Überzeugungen bildeten die

57 Vgl. zur Rektoratsführung Mayers: Anne Chr. NAGEL, Zwischen Führertum und Selbstverwaltung. Theodor Mayer als Rektor der Marburger Universität 1939-42, in: Winfried F. SPEITKAMP (Hg.), Staat, Gesellschaft, Wissenschaft, Marburg 1994, S. 343-364.

58 Theodor MAYER, Deutschland und Europa (Marburger Universitätsreden, 3), Marburg 1940, S. 6.

59 Ibid., S. 21 
ideologische Grundlage für Mayers Vorschläge zur konkreten Umsetzung dieser "gesamteuropäischen Geschichtsbetrachtung", die er in einem Zeitungsartikel vom Februar 1941 präsentierte. "Bei der Geschichtswissenschaft«, schrieb er dort, wist ein gewaltiger Aufstieg seit einiger Zeit in methodischer Hinsicht, aber auch in bezug auf die Darstellung und die Problematik ganz unverkennbar. Aber diese Tatsache fordert, daß ein Gesamtziel und ein Gesamtplan für die Wissenschaftsaufgaben festgelegt wird ${ }^{60}$.

$\mathrm{Daß}$ diese Vorstellungen auch in Mayers wissenschaftsorganisatorische Tätigkeit einflossen, läßt sich an zwei Beispielen zeigen: einerseits an seiner Leitung der Abteilung Mittelalter des Kriegseinsatzes der Geisteswissenschaften, anderseits an seiner Amtsführung als Präsident des Reichsinstituts für ältere deutsche Geschichtskunde, der Monumenta Germaniae Historica.

In seiner Funktion als Leiter des Kriegseinsatzes organisierte Mayer von 1940 bis Kriegsende mehrere Tagungen, zu denen er jeweils eine begrenzte Zahl von Wissenschaftlern verschiedener Fachdisziplinen einlud. Mehrere Publikationen $^{61}$ sowie die überlieferten Protokolle dokumentieren die vielfältigen politischen Implikationen dieser mittlerweile gut erforschten Gemeinschaftsarbeit ${ }^{62}$. Der Landeshistoriker Walter Schlesinger, der damals selbst an einer dieser Tagungen teilgenommen hatte, lobte Mayer nach dem Krieg als außergewöhnlichen Wissenschaftsorganisator, der mit den sogenannten »Gespräche[n] im kleinen Kreise « geradezu revolutionär anmutende Veränderungen im Rahmen der deutschen Mittelalterforschung bewirkt habe ${ }^{63}$. Wirft man jedoch einen Blick auf die erhaltenen Tagungsprotokolle, vor allem aber auf

60 Theodor MAYER, Politik und Geschichte. Aufgaben der Forschung im neuen Europa, in: Berliner Börsenzeitung, 7.2.1941, S. 1f. Dieser programmatische Artikel wurde mehrmals abgedruckt; vgl. Die deutsche Geschichtswissenschaft und ihre Aufgaben im neuen Europa, in: Deutscher Wissenschaftlicher Dienst 33 (9.2.1941); sowie: Die Geschichtsforschung im neuen Europa, in: Völkischer Beobachter, Süddeutsche und Münchner Ausgabe, $11 . / 12.4 .1942$, S. 4.

${ }^{61}$ Fritz HaRTUng, Theodor MaYer, Walter PlatZhoff, Paul RitTERBusCh, Fritz Rörig, Carl SCHMITT, Hans ÜBERSBERGER, Hans ZEISS (Hg.), Das Reich und Europa, Leipzig 1941; Theodor MAYER (Hg.), Adel und Bauern im deutschen Staat des Mittelalters, Leipzig 1943.

62 Vgl. Frank-Rutger HausmanN, »Deutsche Geisteswissenschaft« im Zweiten Weltkrieg. Die »Aktion Ritterbusch« (1940-1945), Dresden, München 1998, S. 201, der resümiert, "daß diese Wissenschaft kontextualisiert war und ideologischen Zwecken diente«. Vgl. über den Stellenwert der Gemeinschaftsarbeit im Leben und Werk Theodor Mayers demnächst Reto HEINZEL, Von der Volkstumswissenschaft zum Konstanzer Arbeitskreis. Theodor Mayer und die interdisziplinäre deutsche Gemeinschaftsforschung, in: Ralph Melville, Stefan AlBRECHT, Jiři MALír (Hg.), Die »sudetendeutsche Geschichtsschreibung« 1918-1960. Zur Vorgeschichte und Gründung der Historischen Kommission der Sudetenländer zur böhmisch-mährischen Geschichte, München 2007 (im Druck).

${ }^{63} \mathrm{Vgl}$. Walter SCHLESINGER, Theodor Mayer und der Konstanzer Arbeitskreis, in: Theodor Mayer und der Konstanzer Arbeitskreis. Theodor Mayer zum 80. Geburtstag, Konstanz 1963, S. 11-29, hier S. 16. 
die einschlägige Korrespondenz, wird klar, daß es für Mayer beim Kriegseinsatz um mehr ging als um die Aufrechterhaltung des wissenschaftlichen Austausches in einer schweren Zeit. Auch sah er darin nicht nur eine momentane, sondern eine dauerhafte Aufgabe. In einem Brief von 1941 beispielsweise zeigte er sich zuversichtlich, daß diese »offizielle Einrichtung [...] wohl nach dem Kriege bleiben [...] und eine wichtige Organisation der Wissenschaft bilden wird “" ${ }^{64}$. Man darf vermuten, daß Theodor Mayer nicht zuletzt an ein Instrument zur Forschungslenkung dachte oder in der Einrichtung zumindest eine Möglichkeit sah, einen substantiellen Beitrag zur Festigung >gesamteuropäischer〈 Forschungsaufgaben zu leisten.

Mit der Übernahme der Leitung der MGH im Frühjahr 1942 erweiterte sich der Spielraum zur Umsetzung von Theodor Mayers wissenschaftsorganisatorischen Plänen. Obschon er in der Nachkriegszeit unablässig beteuerte, er sei zur Übernahme der MGH praktisch gezwungen worden, läßt sich aus den zeitgenössischen Quellen auch etwas anderes herauslesen. "Die Aufgabe ist sehr groß und schön«, schrieb er etwa an seinen Studienfreund August von Loehr, »da ich gleichzeitig den Einsatz der Geisteswissenschaften leite. Dank dieser Doppelfunktion habe er jetzt auch große Möglichkeiten, wie sie vorher nicht bestanden ${ }^{65}$.

Tatsächlich plädierte Mayer in einer Denkschrift, die er im Herbst 1942 ans zuständige Ministerium richtete ${ }^{66}$, für eine grundlegende Änderung im Aufbau und Arbeitsprogramm der MGH. Als oberste Richtlinie schwebte ihm »die Ausrichtung der Arbeiten im volksdeutschen und gemeingermanischen Sinn vor, d.h. die Leistungen der Germanen, besonders aber des deutschen Volkes in Europa« sollten zum Gegenstand der Forschung gemacht werden. Angesichts der weiträumigen Verbreitung germanischer Völker und dem damit kaum zu überblickenden Ganzen mußte dieses Arbeitsprogramm zwangsläufig $z u$ einer "räumliche[n] Ausweitung des Arbeitsgebietes sowie zu einer Konzentrierung des Arbeitseinsatzes auf bestimmte Aufgaben und Probleme« führen. Mayer sprach jetzt von einer "gesamtgermanische[n] Geschichtsbetrachtung", welche das übergeordnete Ziel darstellte. Diese Vorstellung fußte auf der Doktrin, daß eine "gesamteuropäische Betrachtung der europäischen Geschichte des Mittelalters und auch der Neuzeit [...] immer germanisch sein« könne und müsse ${ }^{67}$.

Die von Mayer angestrebte Neuausrichtung der MGH sollte durch die Gründung von »Institute[n] oder Zweigstellen außerhalb des Reiches« flan-

64 StdA Singen, NL Karl Siegfried Bader, Allg. Korrespondenz, Mayer, Theodor, Mayer an Bader vom 12.9.1941.

${ }_{65}$ StdAK, NL Mayer, 13/85, Mayer an Loehr vom 26.4.1942.

${ }^{66}$ BABL, R 4901/14048, Bl. 170, Mayer an REM vom 9.10.1942.

${ }^{67}$ StdAK, NL Mayer, Varia 43, Denkschrift Mayers über Stand und Aufgaben der MGH (o.D.). 
kiert werden. Als Vorbild diente Mayer das Deutsche Historische Institut in Rom, das sich seiner Meinung nach mittlerweile allerdings zu sehr "von seiner Verbindung mit der deutschen Geschichtswissenschaft gelöst« habe ${ }^{68}$. Dem MGH-Präsidenten schien es deshalb notwendig, das Deutsche Historische Institut in Rom, das dem Präsidenten der MGH direkt unterstellt war, »stärker als bisher in den Gesamtaufgabenkreis des Reichsinstituts« einzugliedern. Es sollte nicht länger Papstgeschichte oder italienische Geschichte betreiben, sondern »die germanische und deutsche Geschichte in Italien« pflegen. Die Gründung einer entsprechenden Einrichtung in Paris erhielt in diesem Programm oberste Priorität. Mayer schloß zudem nicht aus, in Zukunft »einen ähnlichen wissenschaftlichen Stützpunkt auch in Madrid zu errichten, um die Germanenforschung dort ins Leben zu rufen ${ }^{69}$.

\section{Fazit}

Theodor Mayers Idee, in Paris ein deutsches historisches Institut ins Leben zu rufen, ist untrennbar mit der Kriegsentwicklung in Westeuropa verbunden. Ohne die französische Niederlage von 1940 wäre ein derartiger Plan nicht denkbar gewesen. Ihm lag die politisch motivierte Vorstellung zugrunde, die deutsche Geschichtswissenschaft habe im nationalsozialistisch beherrschten Europa eine wichtige Aufgabe zu erfüllen. Ziel war es, eine den vermeintlichen Bedürfnissen der deutschen Forschung entsprechende gesamteuropäische Geschichtsbetrachtung zu etablieren.

Zweifelsohne haben Mayers angesehene Position als Rektor der Universität Marburg und späterer MGH-Präsident sowie als Leiter der Abteilung Mittelalter des "Kriegseinsatzes der Geisteswissenschaften« die Aufnahme seiner Vorschläge positiv beeinflußt. Allerdings können die mit dem Plan verbundenen Zielsetzungen nicht allein aus der unmittelbaren Kriegssituation heraus erklärt werden. Vielmehr zeigen sich in seiner Initiative spezifische Denkfiguren, die für den Historiker bereits in der Vergangenheit eine wichtige Rolle gespielt hatten. Der Blick auf Mayers Tätigkeit in den 1930er Jahren macht dabei deutlich, daß er, der über außergewöhnliche Fähigkeiten als Wissenschaftsorganisator verfügte, konkrete wissenschaftspolitische Ziele anvisierte. Seine Befähigung, gepaart mit einem starken Willen sowie einem ausgeprägten Selbstbewußtsein, schufen erst die notwendigen Voraussetzungen dafür, daß Mayer mit seinen Ideen in den gesellschaftlich und politisch spezifischen

69 StdAK, NL Mayer, Varia 43, Denkschrift Mayers über Stand und Aufgaben der MGH (o.D.). 
Bedingungen des nationalsozialistischen Staates Gehör fand, ein Staat, dessen Politik er schon bald nach dem 30. Januar 1933 weitgehend befürwortete.

Auf wissenschaftlicher Ebene war Theodor Mayer von den Methoden und Fragestellungen der deutschen Landes- und Volksforschung derart überzeugt, daß er offen von ihrer Überlegenheit sprach. Dieses Wissenschaftsverständnis basierte auf der Idee nationaler Konkurrenz, allenfalls ging es um die Beeinflussung der ausländischen Forschung. Kooperation und Verständigung hingegen waren diesem Denken fremd. Dies erklärt auch, weshalb Mayer so wenig Interesse an einer Zusammenarbeit zwischen deutscher und französischer Geschichtswissenschaft zeigte ${ }^{70}$. Der Gedanke an eine Institutsgründung in Paris blieb somit bis zum Schluß mit den nationalsozialistischen Vorstellungen einer europäischen Neuordnung gekoppelt.

Das Projekt eines historischen Auslandsinstituts hat Theodor Mayer nach 1945 keineswegs begraben, allerdings ließ sich angesichts der veränderten politischen Lage nicht mehr direkt an die Denkschrift von 1941 anknüpfen. Mayer betonte jetzt, daß die wissenschaftliche und geschichtspolitische Notlage Deutschlands die Etablierung eines neuen krisenfesten Geschichtsbildes, aber auch die Errichtung weiterer Forschungsinstitute, dringend erforderlich machten ${ }^{71}$. Und rund zehn Jahre nach seiner ursprünglichen Denkschrift forderte der Historiker ${ }^{72}$ erneut die Errichtung eines deutschen historischen Instituts in Paris: Abendländische Geschichte setze voraus, schrieb er in einer Rezension, "daß sich der Forscher und Geschichtsschreiber auf eine höhere Warte zu erheben vermag, von der aus er alle Länder gleichmäßig überblickt. Die deutsche Geschichtswissenschaft braucht ein historisches Institut in Paris als Gegenstück zu dem in Rom « ${ }^{73}$.

Angesichts der bevorstehenden Gründung des Centre allemand de recherches historiques stilisierte sich Theodor Mayer in einem Brief an Eugen Ewig zum geistigen Urheber dieser Einrichtung ${ }^{74}$. Daß der Leiter des Konstanzer Ar-

${ }^{70}$ Mayers zu keinem Zeitpunkt näher ausgearbeiteter Vorschlag, es solle ein »ständiger Gedankenaustausch « und eine »ununterbrochene Fühlungnahme « mit der französischen Geschichtswissenschaft herbeigeführt werden, vermag an diesem Befund nichts zu ändern; vgl. Archiv der MGH, 537/63f., Denkschrift über die Errichtung eines deutschen historischen Instituts in Paris vom 10.2.1941.

7 Vgl. über die in der Nachkriegszeit wiederholt gemachten Aufforderungen Mayers zur Errichtung eines krisenfesten Geschichtsbildes HEINZEL, Von der Volkstumswissenschaft zum Konstanzer Arbeitskreis (wie Anm. 61).

72 Obgleich Theodor Mayer als »Mitläufer« entnazifiziert wurde, wurde er im Zuge der Neuorganisation der MGH im Herbst 1947 als Präsident abgesetzt. Vgl. zu diesen Vorgängen: Winfried SCHUŁZE, Deutsche Geschichtswissenschaft nach 1945, München 1989 , S. 150-155

73 Theodor MAYER, Das Hochmittelalter in neuer Schau, in: HZ 171 (1951), S. 449-472, hier S. 471. Darin setzte sich Mayer mit Friedrich Heers umstrittenem Buch »Aufgang Europas« auseinander.

74 StdAK, 10/16, Mayer an Ewig vom 20.11.1957. 
beitskreises damit beide Initiativen in eine gemeinsame geistige Traditionslinie rückte, empfand er ganz offensichtlich nicht als Problem. Dabei hätte er sich darüber im Klaren sein müssen, daß die mit der zukünftigen Forschungsstelle verfolgten Intentionen durchwegs anders gelagert waren als 1941 und $\mathrm{da} ß$ der Gedanke eines Austausches zwischen deutscher und französischer Geschichtswissenschaft erst jetzt eine ernst zu nehmende Rolle spielte. Aus diesen Worten wird ersichtlich, daß sich Theodor Mayer der politischen Dimension und Bedingtheit des Plans von 1941 anscheinend auch in späteren Jahren nicht bewußt war. 
